\title{
ADOLESCÊNCIA E ANTICONCEPÇÃO \\ 1. ESTUDO DE CONHECIMENTO E USO EM PUÉRPERAS INTERNADAS POR PARTO OU ABORTO
}

\author{
Néia Schor* \\ Fanny Lopez A.*
}

\begin{abstract}
SCHOR, N. \& LOPEZ A., F. Adolescência e anticoncepção. 1 - Estudo de conhecimento e uso em puérperas internadas por parto ou aborto. Rev. Saúde públ., S. Paulo, 24: 506-11, 1990.

RESUMO: São analisados o conhecimento e a utilização de métodos anticoncepcionais por adolescentes. Foram levantados os dados a partir de prontuários médicos e de entrevistas relativos a 78 adolescentes puérperas (parto ou aborto), atendidas em um serviço de obstetrícia do Município de Cotia, SP (Brasil), no período de $1 / 5 / 86$ a 31/7/86. Do total de adolescentes estudadas, $61,5 \%$ tinham algum tipo de conhecimento sobre métodos anticoncepcionais, conhecimento este influenciado por fatores tais como idade, escolaridade, paridade e estado marital. As fontes de informação mais procuradas foram os amigos, os parentes e os parceiros, nesta ordem; as menos procuradas foram os profissionais de saúde. Somente uma em cada dez adolescentes usava algum tipo de anticoncepcional, sendo os mais prevalentes a pilula, o método Ogino-Knauss, preservativos e o coito interrompido. Em 100\% dos casos de utilização destes métodos houve indicação por parte de pessoas do grupo social das adolescentes, sendo os anticoncepcionais adquiridos no comércio, sem nenhum controle de saúde.
\end{abstract}

DESCRITORES: Adolescência. Articoncepção, métodos. Conhecimentos, atítudes e prática.

\section{INTRȮDUÇÃO}

As adolescentes representam uma proporção importante na população mundial ${ }^{5}$. Chama a atenção sua inquietante ignorância sexual nos dias de hoje.

Nas últimas décadas, este grupo etário vem se envolvendo em atividades sexuais em idade cada vez mais precoces, sendo observado um decréscimo tanto na idade média da menarca como na idade média da primeira relação sexual ${ }^{1,9}$, bem como aumento no número médio de parceiros sexuais ${ }^{6}$. Esta modificação aos níveis fisiológico e comportamental, no entanto, não é acompanhada de um suficiente conhecimento que as prepare para a vida familiar, para a vida sexual, sendo flagrante seu conhecimento de métodos anticoncepcionais, o que induz esta população etária a conceitos errôneos sobre sexualidade, tais como: acreditar que não engtavidará se as relações forem esporádicas; considerar-se período infértil, quando este não for o caso, e acreditar que os anticoncepcionais são antinaturais, diminuindo e interferindo no prazer sexual. Além disso, está sempre presente entre as adolescentes o temor de que seus pais venham a descobrir sua vida sexual ${ }^{9,12}$.

A ausência de interação entre educação (incluindo aí a dificuldade de acesso à informação, ao aconselhamento e atendimento médico) e comportamen- to sexual, expõe as adolescentes a uma série de graves conseqüências, entre elas a da gravidez que, segundo a Organização Mundial da Saúde (OMS), é um importante fator de risco neste grupo etário ${ }^{11}$.

Assim, pois, o presente trabalho propõe-se a verificar o nivel de conhecimento e utilização de métodos anticoncepcionais por parte daquelas adolescentes que tiveram uma gravidez e recorreram ao Serviço de Obstetricia da Associação de Cotia, Estado de São Paulo, para a resolução de sua gestação.

\section{MATERIAL E MÉTODOS}

O universo da pesquisa compreendeu todas as adolescentes (78) que ingressaram no Serviço de Obstetrícia da Associação Hospital de Cotia (AHC), para atendimento, no período de $1 / 5 / 86$ a $31 / 7 / 86$, e que tiveram como resultado de sua gestação o parto ou o aborto.

Utilizou-se, no presente estudo, a definição cronológica de adolescente, exarada pelo Comitê de Peritos da $\mathrm{OMS}^{10}$, que corresponde ao indivíduo dentro da faixa etária dos 10 aos 20 anos.

O material de estudo compreendeu os prontuários clínicos e as entrevistas feitas com a população adolescente, objeto da pesquisa durante o período de internação.

\footnotetext{
* Departamento de Saúde Materno-Infantil da Faculdade de Saúde Pública da Universidade de São Paulo - Av. Dr. Arnaldo, 715 - 01255 - São Paulo, SP - Brasil.
} 
As entrevistas foram conduzidas mediante a utilização de um formulário, que contemplava as seguintes variáveis: escolaridade; conhecimento, uso e tipo de anticoncepcional; fontes de informação; meios de aquisição dos anticoncepcionais e controle dos mesmos.

\section{As variáveis foram assim definidas:}

- idade atual da adolescente e idade por ocasião da menarca, em anos completos;

- escolaridade: em anos cursados, completos;

- estado marital: (a) estável, quando a adolescente referia morar com o parceiro há um ano ou mais, independente do estado civil ${ }^{6}$; (b) não estável, quando a adolescente referia não morar com um companheiro, independente de seu estado civil;

- conhecimento de métodos anticoncepcionais: classificado de acordo com a indicação espontânea de algum tipo de método;

- uso de métodos anticoncepcionais: classificado conforme a indicação de utilização de um ou outro método, referida pela adolescente.

A tabulação dos dados foi processada através do sistema SPSS (Statistical Package for the Social Science).

\section{RESULTADOS E DISCUSSÃO}

Analisando-se os resultados obtidos, observa-se que a idade média das adolescentes do grupo de estudo foi 17,5 anos. $O$ fato adquire maior relevância se considerarmos que a idade média da população de adolescentes estudada, por ocasião da menarca - expressivo indicador de crescimento e maturação feminina humana, por seu conteúdo biológico, social e psíquico 8 - , girou em torno de 12,3 anos. Estes dados são semelhantes aos encontrados por outros autores em nosso meio $(13,8)^{3}$ mostrando uma tendência de redução na idade da instalação da menarca, com o decorrer dos anos, o que implica novos riscos, entre outros, o da gravidez precoce. $\mathrm{Na}$ população estudada, este evento se deu a partir dos 13 anos, indicando, assim, a necessidade de um maior conhecimento e conscientização quanto às conseqüências do exercício da sexualidade, por parte desta população específica que ingressa na puberdade.

Tais conhecimentos devem abranger o "como", o "quando" e o "onde" da utilização dos diferentes métodos anticoncepcionais disponíveis e, mais que isso, o acesso aos mesmos. Na população de estudo, $61,5 \%$ das adolescentes relataram conhecer pelo menos um método anticoncepcional.

Em relação a algumas variáveis que influem na aquisição de conhecimentos, observa-se, na Fig. 1, que o conhecimento sobre métodos anticoncepcionais foi influenciado diretamente pela variável idade: adolescentes de 13 e 14 anos informaram não conhecer nenhum método anticoncepcional $(0,0 \%)$, enquanto as de 19 anos $(76,0 \%)$ foram as que demostraram maior conhecimento. Estes dados parecem indicar que é apenas nas idades finais que as adolescentes estudadas se encontraram mais receptivas à incorporação de mudanças biopsicossociais, em especial às que se referem à sua sexualidade e a de seu grupo. Pode-se dizer que a quase totalidade dessas adolescentes não está sendo assistida em suas necessidades biopsicossociais por parte da saúde pública.

Relacionando-se escolaridade e nível de conhecimento (Fig. 2), observa-se que as adolescentes relataram conhecer pelo menos um método anticoncepcional; o nível de conhecimento aumentou diretamente à medida em que aumentaram os anos de escolaridade. Poder-se-ia supor que tal fato pudesse decorrer de uma maior possibilidade de acesso aos diferentes meios de informação propiciados pela instrução formal. Contudo, esta hipótese não foi totalmente confirmada: identificou-se um grupo de puérperas sem nenhuma escolaridade, mas com um alto nível de conhecimento $(80,0 \%)$. Além do mais, durante as entrevistas, observou-se que este grupo apresentava uma maior "curiosidade" pelo tema em questão. Buscando-se uma possível explicação para este fato, a maior relação encontrada foi com a variável idade: dentro deste grupo, $85,7 \%$ tinham entre 18 e 19 anos. Este achado leva a crer que esta variável (idade) tem grande influência quanto à aquisiçăo de conhecimentos sobre métodos anticoncepcionais por parte das adolescentes e que a escolaridade, por si so, não é garantia de conhecimentos nesta área específica.

Em relação à variável paridade, a maioria da população $(71,8 \%)$ era composta de primigestas, $o$ que seria de se esperar de uma população jovem; as

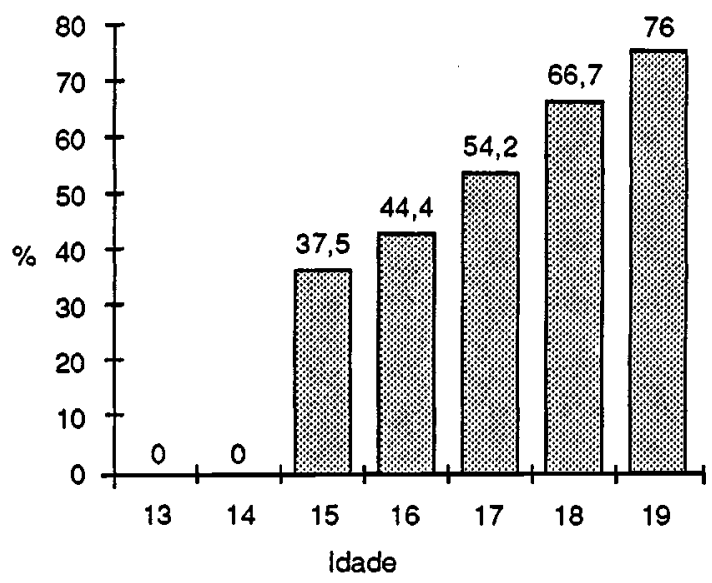

Fig. 1 - "Conhecimentos" sobre métodos contraceptivos, em adolescentes puérperas internadas por parto ou aborto. 


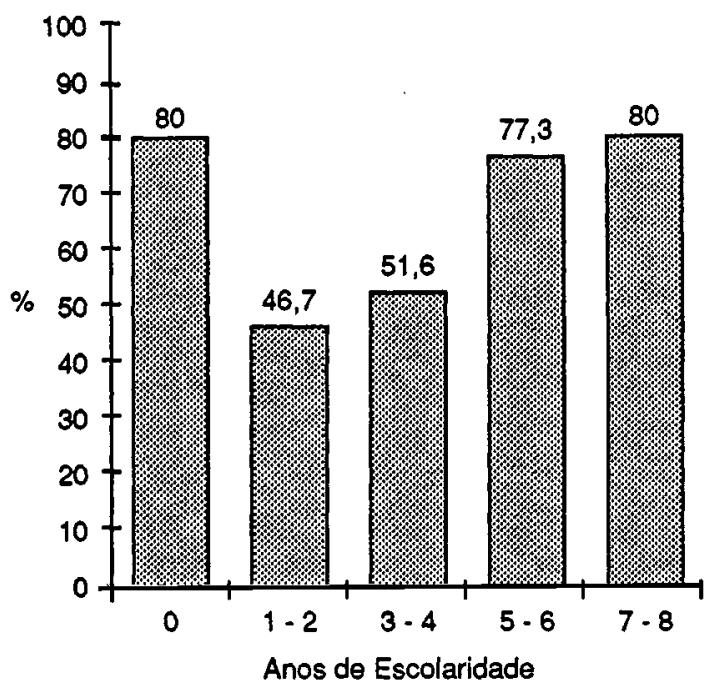

Fig. 2 - Escolaridade e "conhecimentos" sobre métodos contraceptivos, em adolescentes puérperas e internadas por parto ou aborto.

secundigestas perfaziam $25,6 \%$ da população. Os dados mostraram proporções similares de conhecimento sobre métodos anticoncepcionais nos grupos de primigestas $(57,9 \%)$ e secundigestas $(64,7 \%)$. $O$ fato de as adolescentes terem passado por mais de uma experiência com os serviços de saúde, não garantiu maior aquisição de conhecimento, embora se saiba que um dos objetivos programáticos da assistência integral à saúde da mulher é o desenvolvimento das atividades de planejamento familiar ${ }^{7}$.

Quanto à relação entre estado marital e conhecimento, os dados mostraram que as mulheres maritalmente estáveis, que teoricamente "são as que estão mais expostas ao risco de gravide $z^{4}$ ", têm uma proporção relativamente menor de conhecimento de métodos anticoncepcionais $(47,8 \%)$ que as maritalmente não estáveis $(61,8 \%)$, o que poderia estar refletindo uma maior preocupação das adolescentes frente ao risco social de uma gravidez fora de uma relação estável, estimulando, assim, um maior interesse e procura de informações a respeito das possíveis alternativas anticoncepcionais para uma vida sexualmente ativa, sem suas conseqüências indesejáveis.

Quanto ao objeto de estudo, a anticoncepção, observa-se na Fig. 3 que os métodos anticoncepcionais modernos (anticoncepcionais orais, preservativos, injetáveis, DIU e espermicidas) são mais conhecidos que os métodos anticoncepcionais tradicionais (Ogino-Knauss e coito interrompido).

Ainda na Fig. 3, o método anticoncepcional mais referido foi a pílula $(59,2 \%)$, o que concorda com os dados apresentados na literatura mundial 2,4 . A este se segue o método Ogino-Knauss $(13,2 \%)$, chamado comumente de "tabelinha" que, para um bom índice de eficácia, exige das adolescentes um bom conhecimento do seu ciclo menstrual. Os menos referidos foram os espermicidas $(3,9 \%)$ e o coito interrompido (1,3\%), métodos estes que, para serem eficazes, exigem um rigoroso cumprimento das instruções de uso.

Com relação a obtenção da informação referente a estes métodos anticoncepcionais, os meios mais procurados (Fig. 4) foram os canais interpessoais de comunicação, tais como: amigos $(40,9 \%)$, parentes $(21,6 \%)$ e parceiros $(13,6 \%)$, sendo que os menos utilizados foram os profissionais da saúde, em es-

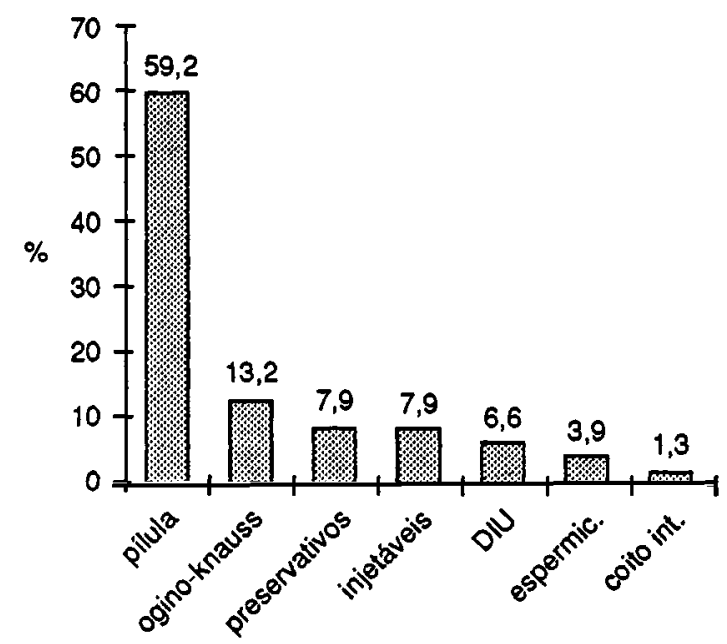

Tipo de Método de Contraceptivo

Fig. 3 - "Conhecimentos" sobre diferentes métodos contraceptivos, em adolescentes puérperas internadas por parto ou aborto.

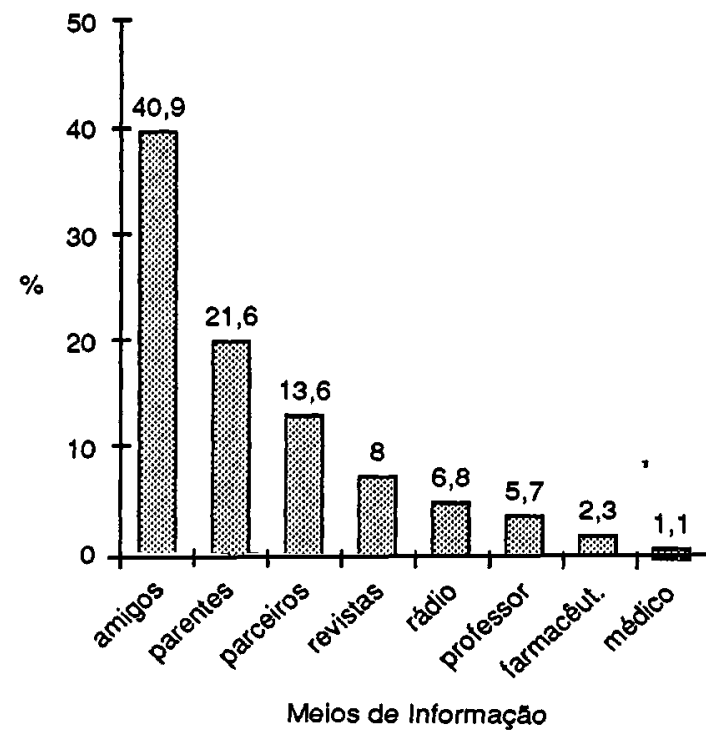

Fig. 4 - "Conhecimentos" sobre métodos contraceptivos, segundo meios de informação, em adolescentes puérperas internadas por parto ou aborto. 
pecial o médico $(1,1 \%)$, revelando a ausência da educação em saúde por parte dos serviços de saúde no que se refere à prática anticoncepcional, o que leva a supor que o meio social daquelas adolescentes foi altamente influente quanto à informação informal.

Sabe-se que em nível comunitário, de uma maneira geral, obtém-se informação incompleta e errônea. Desta forma, os dados do estudo autorizam a formulação da hipótese de que uma utilização adequada destes canais interpessoais de comunicação, através de uma boa orientação e treinamento dos líderes naturais da comunidade, aumentaria em quantidade e em qualidade os índices de conhecimento de métodos anticoncepcionais.

Segundo a Pesquisa Mundial da Fertilidade, 1980, "o uso de métodos anticoncepcionais está regulado principalmente pelo conhecimento adequado, incluindo a forma correta de utilização e a disponibilidade de elementos em relação a esta prática anticoncepcional ${ }^{4 " .}$.

Observou-se por parte das adolescentes da população em estudo uma grande lacuna entre o índice de conhecimento e o de utilização de métodos anticoncepcionais: $6 \mathrm{em}$ cada 10 adolescentes que tiveram uma gravidez referiram, espontaneamente, conhecer algum tipo de anticoncepcional, mas somente 1 em cada 10 informou ter utilizado algum método. Em relação às adolescentes que terminaram sua gestação em parto, $58,6 \%$ conheciam, mas apenas $24,4 \%$ utilizavam algum método anticoncepcional. Estes dados indicam uma deficiência quanto às necessidades não atendidas desta população exposta a uma gravidez de maior risco.

A utilização do aborto entre a população de adolescentes, com um dos métodos anticoncepcionais, o que não estaria ocorrendo se a lacuna entre conhecimento $(85,5 \%)$ e utilização de métodos anticoncepcionais $(28,6 \%)$ fosse diminuída através da atuação dos serviços de saúde. Uma adequada educação em saúde poderia repercutir favoravelmente nos índices de morbimortalidade materna, relativos a esta faixa etária.

A utilização inadequada de anticoncepcionais por parte de adolescentes sexualmente ativas pode levar a uma série de efeitos colaterais biopsicossociais. Portanto, há que se fazer uma avaliação das indicações e contraindicações de seu uso por adolescentes, acompanhada de uma ampla divulgação dos resultados, como também há que se promover e divulgar um acesso seguro e irrestrito às informações e ao atendimento em saúde.

Na população estudada, a pílula $(45,5 \%)$ foi o método anticoncepcional mais utilizado, seguido dos métodos que não exigem prescrição médica: Ogino-Knauss $(27,2 \%)$, preservativos $(18,2 \%)$ e coito interrompido $(9,1 \%)$.
Observou-se que os anticoncepcionais orais estão sendo mais utilizados pelos jovens em virtude de haver uma maior divulgação quanto ao seu uso, de serem mais eficazes ${ }^{1}$ e de haver maior facilidade de compra, o que não significa que este seja o método mais indicado para esta faixa etária. Do ponto de vista clínico, a anticoncepção oral pode interferir no crescimento físico e na fecundidade futura, em especial quando não se tem estabelecido um padrão menstrual regular.

O método Ogino-Knauss é o que apresenta menor eficácia; para aumentá-la haveria necessidade de uma compreensão de seu uso, de uma atividade sexual programável e de períodos menstruais regulares, o que não é o comum dentro deste grupo etário, caracterizado por períodos menstruais irregulares, atividade sexual esporádica e não planejada, o que leva, na maioria das vezes, a uma gravidez nåo desejada.

Quanto aos preservativos, estes se apresentam como um dos métodos mais apropriados para este grupo estário, tendo-se em vista: o envolvimento do companheiro na iniciativa da anticoncepção; a prevenção de uma gravidez não desejada; a prevenção de doenças sexualmente transmissíveis; não ter, praticamente, efeitos colaterais; facilidade de aquisição e baixo custo. Todos estes fatores fazem deste método o mais adequado para a indicação em programas de saúde da adolescente. Apesar dessas ponderaçóes, observou-se, no presente estudo, que este método não tem sido muito utilizado pelos adolescentes.

Em relação ao coito interrompido, sabemos que para jovens sexualmente inexperientes, este pode ser um método inseguro e de difícil utilização; ele não pode ser descartado, contudo, em relaçōes sexuais não planejadas, que ocorrem com maior freqüência neste grupo etário.

$\mathrm{Na}$ busca de um método anticoncepcional ideal, observou-se que, há uma ampla variedade deles, năo existindo ainda um que seja inócuo, eficaz, acessível, fácil de usar, de baixo custo e que se adapte a todas as necessidades do grupo. Portanto, $a$ indicação de um determinado método anticoncepcional deve ser avaliada em função da relação risco-benefício e não da disponibilidade dos métodos, pelos serviços de saúde.

Um serviço de saúde deveria facilitar a utilização de diversos métodos anticoncepcionais, compreendendo o controle e indicação adequados, a fim de prevenir efeitos colaterais e complicaçoes. $\mathrm{Na}$ população estudada, a totalidade dos métodos anticoncepcionais utilizados foi indicada por terceiros ou comercialmente (100\%) e sem nenhum controle $(100 \%)$, o que revela a não participação dos profissionais e dos serviços de saúde no planejamento reprodutivo da população. 


\section{CONCLUSÕES}

O tema deste trabalho é bastante complexo e apresenta vários fatores de envolvimento.

A pesquisa revela que mais de $1 / 3$ das adolescentes puérperas estudadas não puderam exercer o direito pessoal de ter ou não uma gravidez, pois a opção a esse direito sofreu a influência, sobretudo, da falta de conhecimento de métodos anticoncepcionais.

O nível de conhecimento de métodos anticoncepcionais mostrou-se altamente relacionado com a variável idade, aumentando à medida em que esta se elevava em anos.

Observou-se uma grande lacuna entre conhecimento e utilização de métodos anticoncepcionais entre as adolescentes estudadas, sendo maior entre as que terminaram sua gravidez em aborto.

A totalidade das indicações dos métodos anticoncepcionais e das informações sobre aquisição e utilização dos mesmos foi feita em nível do próprio meio sóciocultural das adolescentes estudadas, e não em nível dos serviços de saúde.

Em função destes resultados, acredita-se que as atividades de planejamento familiar, dentro de um programa de saúde da mulher, com boa motivação e organização, não sofreriam resistência por parte desta população, visto que uma parcela das adolescentes estudadas mostrou-se bastante receptiva a aprender e a mudar seu comportamento nesta área específica.

Um maciço investimento financeiro na área da saúde da mulher (com um atendimento específico a mulheres púberes e adolescentes, atualmente inexistente), que contemplasse aspectos educacionais, médicos e assistenciais, poderia ter efeitos de grande alcance na diminuição da relação custos / benefícios da área de saúde e na elevação da qualidade de vida da mulher, com repercursões positivas para os dias de hoje e para o futuro. As empresas privadas poderiam desempenhar um importante papel através da mídia, financiando a leitura e a apresentação de pequenos programas de educação em saúde, indicando a existência e a localização dos serviços de saúde, tendo seus gastos com essa iniciativa descontados no Imposto de Renda.

\section{AGRADECIMENTOS}

Ao Diretor e à Equipe do Serviço de Obstetrícia da Associação Hospital de Cotia, São Paulo, pela oportunidade oferecida para a realização da presente pesquisa.

SCHOR, N. \& LOPEZ A., F. [Adolescence and contraception. 1 - A study of knowledge and use among women interned for childbirth or abortion]. Rev. Saúde públ., S. Paulo, 24: 506-11, 1990.

ABSTRACT: A study of the knowledge and utilization of contraceptive methods by adolescent is presented. An analysis was carried out based on data collected from interviews with and recorded case histories of 78 puerperal adolescents (childbirth or abortion), assisted by an obstetric service in the county of Cotia, SP, Brazil, between May 1 and July 31, 1986. Of all the adolescents studied, 61.5\% had some knowledge of contraceptive methods; the findings showed that such knowledge was influenced by factors such as: age, school background, parity and marital status. The main sources of information on contraception were: friends, relatives and partners, in this order; those least sought for in this regard were health professionals. Only one in each ten adolescents made use of some contraceptive measure, the most prevalent methods being the contraceptive pill, the Ogino-Knauss method, condoms and coitus interruptus. In al of the cases of the utilization of these methods the same had been "recommended" by persons belonging to the adolescents' social group, and had been acquired in shops, without any health control.

KEYWORDS: Adolescence. Contraception, methods. Knowledge, attitudes, practice. 


\section{REFERENCIAS BIBLIOGRÁFICAS}

1. FRIEDMAN, H. L. \& EDSTROM, K. G. Higiene de la reproduccion en la adolescencia: procedimiento para la planificación de investigaciones sobre servicios de salud. Ginebra, Organizacion Mundial de la Salud, 1983. (OMS - Publicacion Offset, 77).

2. LEVANTAMENTOS sobre fecundidade e planejamento familiar: uma atualização. Popul. Rep. Ser. M., (8) 1987.

3. MARTINS, A. D. Idade da menarca em nosso meio. $J$. bras. Ginec., 90: 77-80, 1980.

4. PESQUISA mundial sobre fecundidade: estudo atual e constatações. Popul. Rep. Ser. M., (3) 1980.

5. ROGEL, M. et al. Contraceptive behavior in adolescence: a decision-making perspective. J.Youth Adolesc., 9: 491-506, 1980.

6. SCHOR, N. Aborto como questâo de saúde pública: estudo da demanda de mulheres que recorreram ao Hospital por complicaçōes do aborto. São Paulo, 1984. [Tese de Doutorado - Faculdade de Sáude Pública da USP].

7. SECRETARIA DE ESTADO DA SAUUDE. Grupo de Saúde da Mulher. Subprograma de saúde da mulher: assistência ao planejamento familiar. São Paulo, 1986. v.5. [Mimeografado].
8. SEDENHO, N. \& SOUZA FREITAS, J. A. Fatores que influenciam a ocorrência da menarca. J. bras. Ginec., 94: $303-8,1984$.

9. SILBER, T. EL embarazo en adolesentes en Estados Unidos de América. In: Organización Panamericana de la Salud. Salud maternoinfantil, atencion primaria en las Américas: hechos y tendencias. Washington, D.C., 1984. p. 232-9. (Publicacion Cientifica, 461).

10. WORLD HEALTH ORGANIZATION. Expert Committee on Health Needs of Adolescent's, Geneva, 1976. Report. Geneva, 1977. (Technical Report Series, 609).

11. WORLD HEALTH ORGANIZATION. Risk approach for maternal and child health care. Geneva, 1978. (Offset Publication, 39).

12. ZELNICK, $M$. et al. Reasons for nonuse of contraception by sexually active women aged 15-19. Fam. Plan. Perspect., 11: 289-96, 1979.

Recebido para publicação em 12/i1/1990 Reapresentado em 10/9/1990 Aprovado para publicação em 20/9/1990 